\title{
Yukawa-SYK model and self-tuned quantum criticality
}

\author{
Gaopei Pan $\odot,{ }^{1,2}$ Wei Wang $\odot,{ }^{1,2}$ Andrew Davis $\odot,{ }^{3}$ Yuxuan Wang $\odot,{ }^{3, *}$ and Zi Yang Meng $\oplus^{1,4, \dagger}$ \\ ${ }^{1}$ Beijing National Laboratory for Condensed Matter Physics and Institute of Physics, Chinese Academy of Sciences, Beijing 100190, China \\ ${ }^{2}$ School of Physical Sciences, University of Chinese Academy of Sciences, Beijing 100190, China \\ ${ }^{3}$ Department of Physics, University of Florida, Gainesville, Florida 32601, USA \\ ${ }^{4}$ Department of Physics and HKU-UCAS Joint Institute of Theoretical and Computational Physics, The University of Hong Kong, \\ Pokfulam Road, Hong Kong SAR, China
}

(Received 30 September 2020; accepted 24 December 2020; published 18 March 2021)

\begin{abstract}
Non-Fermi liquids (NFLs) are a class of strongly interacting gapless fermionic systems without long-lived quasiparticle excitations. An important group of NFL models feature itinerant fermions coupled to soft bosonic fluctuations near a quantum-critical point and are widely believed to capture the essential physics of many unconventional superconductors. However, numerically, the direct observation of a canonical NFL behavior in such systems, characterized by a power-law form in the Green's function, has been elusive. Here, we consider a Sachdev-Ye-Kitaev (SYK)-like model with random Yukawa interaction between critical bosons and fermions (dubbed the Yukawa-SYK model). We show that it is immune from the minus-sign problem and hence can be solved exactly via large-scale quantum Monte Carlo simulation beyond the large- $N$ limit accessible to analytical approaches. Our simulation demonstrates that the Yukawa-SYK model features "self-tuned quantum criticality"; namely, the system is critical independent of the bosonic bare mass. We put these results to the test at finite $N$, and our unbiased numerics reveal clear evidence of these exotic quantum-critical NFL properties-the power-law behavior in the Green's function of fermions and bosons-which propels the theoretical understanding of critical Planckian metals and unconventional superconductors.
\end{abstract}

DOI: 10.1103/PhysRevResearch.3.013250

\section{INTRODUCTION}

The non-Fermi liquid (NFL) is a state of gapless fermionic matter that does not have long-lived quasiparticles due to its strongly interacting nature $[1,2]$. It is widely believed to be the microscopic origin of the "strange metal" state observed in a broad range of materials, such as $\mathrm{Cu}$-based [3] and $\mathrm{Fe}$ based $[4,5]$ high-temperature superconductors, heavy-fermion compounds [6,7], and recently in twisted two-dimensional (2D) heterostructures [8,9]. Additionally, the understanding of the unconventional superconducting phase in these systems naturally hinges on the understanding of the NFL "normal state." Moreover, recently from studies of the Sachdev-YeKitaev (SYK) models [10-13], it has been realized that NFLs host a hidden connection between strange metals [14] and states of holographic quantum matter that saturate the upper bound for quantum chaos, opening an entirely new avenue in understanding the behavior of NFLs [15].

The term "non-Fermi liquid" captures the failure of the conventional perturbative approach in treating interacting fermion systems with weak interactions, which poses a challenge in the theoretical understanding of such systems. In

\footnotetext{
*yuxuan.wang@ufl.edu

†zymeng@hku.hk
}

Published by the American Physical Society under the terms of the Creative Commons Attribution 4.0 International license. Further distribution of this work must maintain attribution to the author(s) and the published article's title, journal citation, and DOI. a general context, NFL behavior often occurs via electron interactions mediated by gapless bosonic modes [16-27] that render the electrons incoherent. Such gapless bosons typically arise in the vicinity of a quantum-critical point (QCP) or in quantum gauge theories. Despite the simplicity of the setup, the analytical solution to these models remains challenging due to the lack of a natural small control parameter. Advances have been made via modifying the model to a large $N$ limit, with $N$ being the number of fermion flavors and with a leap of faith that the same physics holds down to $N=O(1)$, while these large- $N$ approaches face important subtleties in two spatial dimensions [28].

Along a separate path, there has been great progress on the numerical front in recent years, in particular, in a designer Hamiltonian of critical bosons Yukawa coupled to Fermi surfaces $[27,29,30]$. Recent results in minus-sign-problem-free quantum Monte Carlo (QMC) simulations [23-27,30] have shown strong evidence of NFL states in a range of such boson-fermion models with gapless bosons from nematic [31] and ferromagnetic [23,32] quantum-critical points and with gauge fields [26,33,34] (see Ref. [27] for a recent review). It is now possible to obtain accurate and reliable information about the scaling behaviors in the close vicinity of these QCPs, testing and improving our theoretical knowledge about these challenging problems.

To reveal NFL physics in numerics, this class of models require tuning the mass of the boson to a critical value, while away from the QCP the system restores Fermi liquid behavior. However, the precise determination of the quantum-critical point and the region of the NFL are subject to finite-size effects, and the position of the QCP is not universal, but 
system dependent. Moreover, recently it was found that to reveal the clear signature of a NFL in fermion self-energy in these QCP systems, one would also need to control the strength of the effective coupling between fermions and bosons, as well as deduct the non-negligible thermal contributions to the fermionic self-energy [32]. These difficulties make it harder to directly reveal the scaling form of the NFL self-energies in these systems.

Recently, a class of SYK-like models featuring random Yukawa interactions between bosons and fermions has been put forward to analyze the NFL pairing problem [35-38]. Analytically solvable in a large- $N$ limit similar to the SYK models, the "Yukawa-SYK model" takes a different approach from the perturbative one to address the interacting fermion system by eliminating kinetic energies from the outset. Physically, such a theoretical approach is of relevance to systems where the Fermi energy is small, e.g., systems with low electron density such as $\mathrm{SrTiO}_{3}$ and Moiré flat band systems such as twisted bilayer graphene [39-41]. The Yukawa-SYK models have been shown to be maximally chaotic [42] and thus likely to admit a holographic dual description. Compared with the SYK models that only involve interacting fermions, the inclusion of a dynamical bosonic degree of freedom in the Yukawa-SYK model makes it ideal to model strongly interacting fermionic systems near a QCP.

Unlike finite-dimensional models with quantum-critical points, within large- $N$ approximation these models have been shown to "self-tune" to quantum criticality; that is, the system becomes critical due to the strong mutual feedback between the bosonic and fermionic sectors, independent of the bosonic bare mass. In addition, the pairing behavior at large $N$ has been analytically studied [35-37]. Depending on details of the Yukawa coupling, these models show either exotic pairing of incoherent fermions or a NFL phase that is stable to pairing down to $T=0$. While the onset temperature of pairing may be finite, the feedback effects of pairing fluctuations to the fermion Green's function are small $[\sim \mathcal{O}(1 / M N)]$, without affecting the NFL behavior of the normal state.

As in the original SYK model, these analytical results of the NFL behavior are formally obtained by using the replica trick and then taking the replica-diagonal ansatz. This is equivalent to replacing the quenched disorder with annealed disorder, which is usually justified by the fact that replicanondiagonal processes are suppressed by $1 / N$ [15]. However, the validity of this ansatz is far from obvious [43], since it is not clear whether the summation of the subdominant processes, each small in $1 / N$, is convergent. If the system breaks replica symmetry, the true ground state is then a spin glass. For example, replica symmetry breaking occurs in the bosonic SYK model $[44,45]$, and in fact it has been shown recently that similar situations occur for all random interacting bosonic models [43]. On the other hand, for the fermionic SYK model, there is now strong numerical and analytical evidence that a glass phase is absent and the NFL state persists down to $T=0$ [44-46]. For this reason the validity of the large- $N$ analytical result of the Yukawa-SYK model needs to be carefully investigated, especially since the model involves both fermions and bosons. To this end, unbiased numeric calculations, similar in spirit to the aforementioned critical bosons Yukawa coupled to Fermi surface systems [23-27,29$31,33,47]$, are highly desirable.
With this motivation in mind, here we address such a timely issue by showing that at finite $N$ the Yukawa-SYK model can be exactly solved by determinantal QMC (DQMC) simulations, thanks to the bosonic degree of freedom. A simple extension of the original model, introducing an antiunitary time-reversal symmetry, eliminates the minus-sign problem without altering the essential physics. To enable a direct comparison with QMC, we numerically solve the self-energies of the Yukawa-SYK model within large $N$ at finite temperatures with discrete imaginary time steps. At low temperatures, this indeed agrees with the analytical solution of the SchwingerDyson equations with an emergent time-reparametrization symmetry. This emergent symmetry indicates that the effect of thermal fluctuations can be easily incorporated in the time domain (see Refs. [32,48] for subtleties in the frequency domain), enabling a direct identification of the NFL behavior at finite temperatures.

In this paper, we found that as one progressively increases $N$, the Green's functions from QMC simulations do approach the large- $N$ result and display self-tuned criticality and NFL behavior with power-law self-energies. Additionally, we found that as $N$ increases, the QMC result with different realizations of the random interaction self-average (i.e., the variance of the Green's function) decreases with increasing $N$, and we obtained a good match with the large- $N$ results. This is strong evidence that the system is free from glassy behavior at least within the temperature range accessible to QMC. By comparing with large- $N$ results, we analyze the behavior of finite- $N$ corrections and show that it is consistent with those by processes suppressed by $1 / N$, including processes involving replica-off-diagonal fluctuations and pairing fluctuations. By contrast, we consider a model [35] in a similar form with a crucial difference that the random coupling is of a lower rank. In such a model with less randomness, replica-off-diagonal processes are less suppressed. We numerically show that the bosonic Green's function exhibits glassy behavior.

\section{THE MODEL}

The model studied here describes $M$ quantum dots, each hosting $N$ flavors of fermions interacting with $N^{2}$ flavors of matrix bosons via all-to-all random Yukawa interactions. The Hamiltonian of this Yukawa-SYK model is given by

$$
\begin{aligned}
H= & \sum_{i, j=1}^{M} \sum_{\alpha, \beta=1}^{N} \sum_{m, n}^{\uparrow, \downarrow}\left(\frac{i}{\sqrt{M N}} t_{i \alpha, j \beta} \phi_{\alpha \beta} c_{i \alpha ; m}^{\dagger} \sigma_{m, n}^{z} c_{j \beta ; n}\right) \\
& +\sum_{\alpha, \beta=1}^{N}\left(\frac{1}{2} \pi_{\alpha \beta}^{2}+\frac{m_{0}^{2}}{2} \phi_{\alpha \beta}^{2}\right),
\end{aligned}
$$

where the random coupling between fermion and boson satisfies $\quad\left\langle t_{i \alpha, j \beta}\right\rangle=0, \quad\left\langle t_{i \alpha, j \beta} t_{k \gamma, l \delta}\right\rangle=$ $\left(\delta_{\alpha \gamma} \delta_{i k} \delta_{\beta \delta} \delta_{j l}+\delta_{\alpha \delta} \delta_{i l} \delta_{\beta \gamma} \delta_{j k}\right) \omega_{0}^{3}$. This model is very similar to that studied in Ref. [35], the only difference being that here the random coupling $t_{i \alpha, j \beta}$ has a higher rank than that in Ref. [35] that does not depend on $\alpha$ and $\beta$. As we will see in Sec. III, the high-rank randomness of the Yukawa coupling $t_{i \alpha, j \beta}$ is crucial for stabilizing the non-Fermi liquid behavior. $\pi_{\alpha \beta}$ is the canonical momentum of $\phi_{\alpha \beta}$. Hermiticity of the first term requires $\phi_{\alpha \beta}=-\phi_{\beta \alpha}$. As schematically 


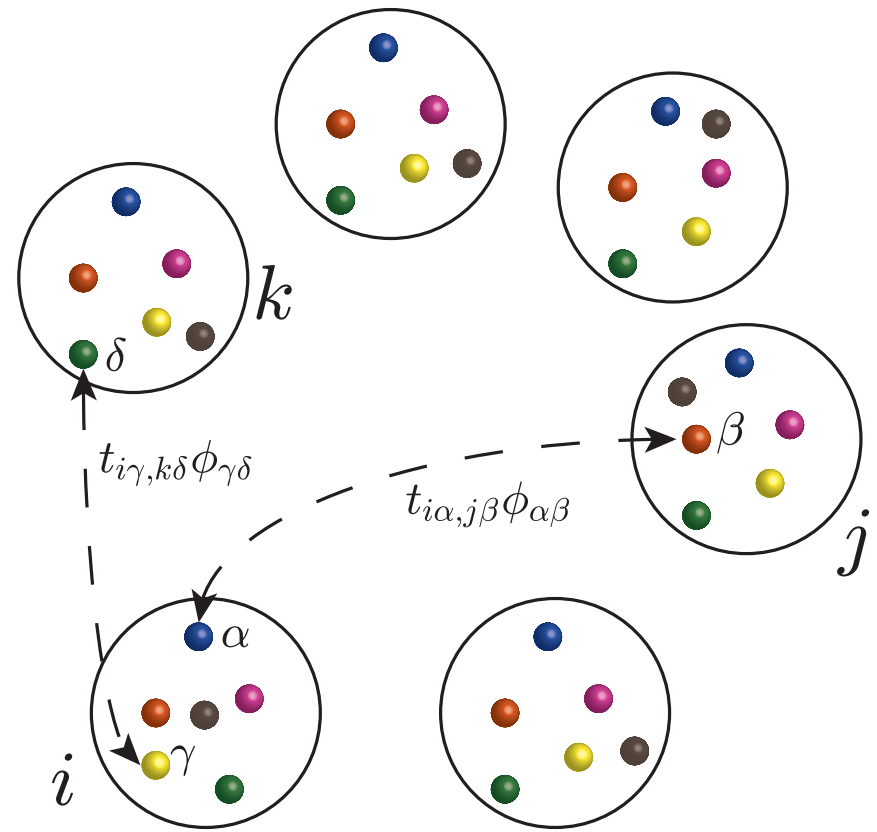

FIG. 1. Yukawa-SYK model. There are $M$ quantum dots labeled by $\{i, j\}$, and each dot has $N$ flavors labeled by $\{\alpha, \beta\}$. Bosons are given by antisymmetric fields $\phi_{\alpha \beta}$. Fermions are coupled to bosons through a random Yukawa coupling $t_{i \alpha, j \beta}$.

depicted in Fig. 1, here $(\alpha, \beta)$ are flavor indices, and $(i, j)$ are site indices. $\sigma^{z}$ is the Pauli matrix in the fermion spin space for each flavor. In the absence of a chemical potential term $\mu=0$, the model has an exact particle-hole symmetry under $c \rightarrow c^{\dagger}$. The general case with $\mu \neq 0$ has also been recently analytically solved at the $N, M \rightarrow \infty$ limit [38], and numerical studies are needed. Importantly, this model has a time-reversal symmetry $c^{\dagger} \rightarrow c^{\dagger} i \sigma^{y} \mathcal{K}$, where $\mathcal{K}$ is the complex conjugation operator, which guarantees the absence of the minus-sign problem of the QMC simulation. For the sake of simplicity we set $\omega_{0}=1$ as the energy unit throughout the paper. The only other energy scale in Eq. (1) is the bosonic bare mass $m_{0}$. We refer to situations with small and large $\omega_{0} / m_{0}$ as "weakly coupled" and "strongly coupled."

In the $N \rightarrow \infty, M \rightarrow \infty$ limit, the ground state of the system has been analytically solved [35,36], and the ground state is found to be a non-Fermi liquid. The large- $N$ result is based on the assumption that the replica symmetry of the random model is unbroken. In this paper we verify the validity of the non-Fermi liquid solution by examining and extrapolating the system behavior at finite $N, M$. For such $N, M$, analytical calculations are uncontrolled. Fortunately, due to the time-reversal symmetry in our designer Hamiltonian in Eq. (1) there is no minus-sign problem (the proof of this is given in Appendix A 3).

\section{A. Normal-state results at $N, M \rightarrow \infty$}

Before demonstrating our QMC results for the YukawaSYK model, we first briefly review the theoretical results at the $N, M \rightarrow \infty$ limit. In this limit one can show that the effective action has a saddle point given by the Schwinger-Dyson equations

$$
\begin{aligned}
& \Pi\left(i \Omega_{n}\right)=\frac{4 M}{N} \omega_{0}^{3} T \sum_{m} G_{f}\left(i \omega_{m}-i \Omega_{n} / 2\right) G_{f}\left(i \omega_{m}+i \Omega_{n} / 2\right), \\
& \Sigma\left(i \omega_{m}\right)=-\omega_{0}^{3} T \sum_{m} G_{b}\left(i \Omega_{n}\right) G_{f}\left(i \omega_{m}-i \Omega_{n}\right),
\end{aligned}
$$

where $\Sigma$ and $\Pi$ are fermionic and bosonic self-energies, respectively, and $G_{f}\left(i \omega_{m}\right)=\left[i \omega_{m}+\Sigma\left(i \omega_{m}\right)\right]^{-1}$ and $G_{b}\left(i \Omega_{n}\right)=$ $\left[\Omega_{n}^{2}+\Pi\left(i \Omega_{m}\right)+m_{0}^{2}\right]^{-1}$ are fermionic and bosonic Green's functions, respectively.

At $T=0$, it was found [35,36] that for $m_{0} \sim \omega_{0}$ and $\omega, \Omega \ll \omega_{0}$ the self-energies are given by

$$
\begin{aligned}
& \Sigma(\omega)=-G_{f}(\omega)^{-1}=i c \operatorname{sgn}(\omega)|\omega|^{x} \omega_{0}^{1-x}, \\
& \Pi(\Omega)=G_{b}(\Omega)^{-1}=-m_{0}^{2}+c^{-2} \alpha(x)|\Omega|^{1-2 x} \omega_{0}^{1+2 x},
\end{aligned}
$$

where $c$ is a nonuniversal $O(1)$ constant, and $0<x<1 / 2$ is determined by

$$
\frac{4 M}{N}=\frac{1 / x-2}{1+\sec (\pi x)}
$$

and

$$
\alpha(x)=-\frac{\Gamma^{2}(-x)}{4 \pi \Gamma(-2 x)} .
$$

Compared with the results in Ref. [35], Eq. (4) is different by a factor of 2 because of the addition of the spin degree of freedom $m, n=\uparrow / \downarrow$ in the Hamiltonian in Eq. (1). In particular, at $M=N$, one finds $x \approx 0.098$, and for $4 M=N$, $x \approx 0.231$.

From Eq. (3) we have

$$
m_{0}^{2}-\Pi(\Omega=0)=0,
$$

indicating that the boson is critical. This was argued in Refs. [35,36] to be true for an arbitrary $m_{0}^{2}$. No matter what the bosonic bare mass is, the system renormalizes it to zero via interaction effects. For this reason we dub this phenomenon "self-tuned quantum criticality." This feature is certainly not present in any finite-dimensional models such as those of critical bosons coupled to Fermi surface systems [16-27,30 $33,47,49]$ discussed in Sec. I.

In the time domain, by a Fourier transform we obtain [50]

$$
\begin{aligned}
\Pi(\tau, \tilde{\tau}) & \propto|\tau-\tilde{\tau}|^{-(2-2 x)}, \\
G_{b}(\tau, \tilde{\tau}) & \propto|\tau-\tilde{\tau}|^{-2 x}, \\
\Sigma(\tau, \tilde{\tau}) & \propto|\tau-\tilde{\tau}|^{-(1+x)} \operatorname{sgn}(\tau-\tilde{\tau}), \\
G_{f}(\tau, \tilde{\tau}) & \propto|\tau-\tilde{\tau}|^{x-1} \operatorname{sgn}(\tau-\tilde{\tau}) .
\end{aligned}
$$

It is known that at a finite but low temperature $T=1 / \beta \ll$ $\omega_{F}$ [10-13], the long-time correlated behavior persists, and it was found $[35,36]$ that for the present model $\omega_{F} \equiv \omega_{0}^{3} / m_{0}^{2}$. One can accordingly obtain the fermionic and bosonic Green's functions through a reparametrization symmetry transformation $\tau \rightarrow f(\tau)=\tan (\pi \tau / \beta)$ [10-13], and we have, at low temperatures and long-time limit,

$$
\begin{aligned}
& G_{f}(\tau, 0) \propto\left(\frac{\pi}{\beta \sin (\pi \tau / \beta)}\right)^{1-x}, \\
& G_{b}(\tau, 0) \propto\left(\frac{\pi}{\beta \sin (\pi \tau / \beta)}\right)^{2 x} .
\end{aligned}
$$



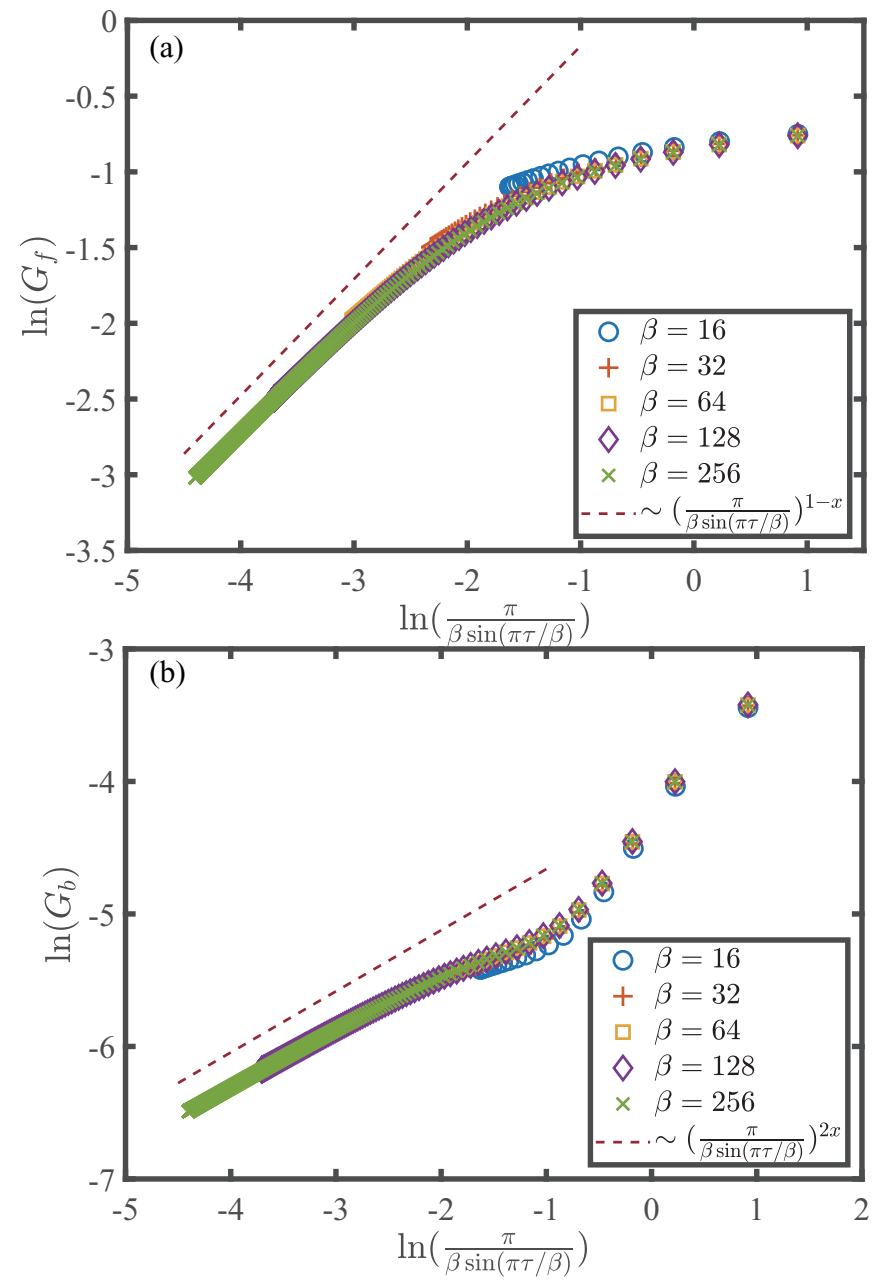

FIG. 2. Theoretical result of (a) $G_{f}$ and (b) $G_{b}$ at $N=4 M \rightarrow \infty$, $\omega_{0}=1, m_{0}=2$, and various temperatures. Here, we show them in $\log$-log plots. The auxiliary dashed lines, whose slopes are $1-x$ and $2 x$, respectively, show that $G_{f}(\tau, 0) \propto\left(\frac{\pi}{\beta \sin (\pi \tau / \beta)}\right)^{1-x}$ and $G_{b}(\tau, 0) \propto$ $\left(\frac{\pi}{\beta \sin (\pi \tau / \beta)}\right)^{2 x}$ at $\tau \rightarrow \frac{\beta}{2}$, when $\beta$ is large enough.

To enable a direct comparison with the QMC data, we developed an iterative algorithm to solve the nonlinear equation in (2) numerically at an arbitrary temperature. To ensure the convergence of the iterations, the temperature dependence of $\Pi(0)$ was fixed using analytical results obtained in Ref. [36]. As we shall see, the QMC simulations for the bosonic sector are performed on a time lattice with lattice constant $\Delta \tau$, and the Matsubara frequencies are compact and defined in a frequency Brillouin zone $\omega_{m}, \Omega_{n} \in(-\pi / \Delta \tau, \pi / \Delta \tau)$. We have incorporated the compactness of the frequency domain within our numerical solution of Eq. (2) as well, which ensures a better match with QMC results especially at large frequencies.

In Fig. 2 we plot the behavior of $G_{f}$ and $G_{b}$ at $N=4 M$, $\omega_{0}=1, m_{0}=2$, and different temperatures from iterative theoretical calculation; in particular, we see that at $\beta=256$ $\left(T=\frac{1}{256}\right)$, the results match well in the long-time limit with the approximate result obtained using time-reparametrization symmetry, exhibiting self-tuned criticality and NFL behaviors. This result will be compared with numerical ones in Sec. III.

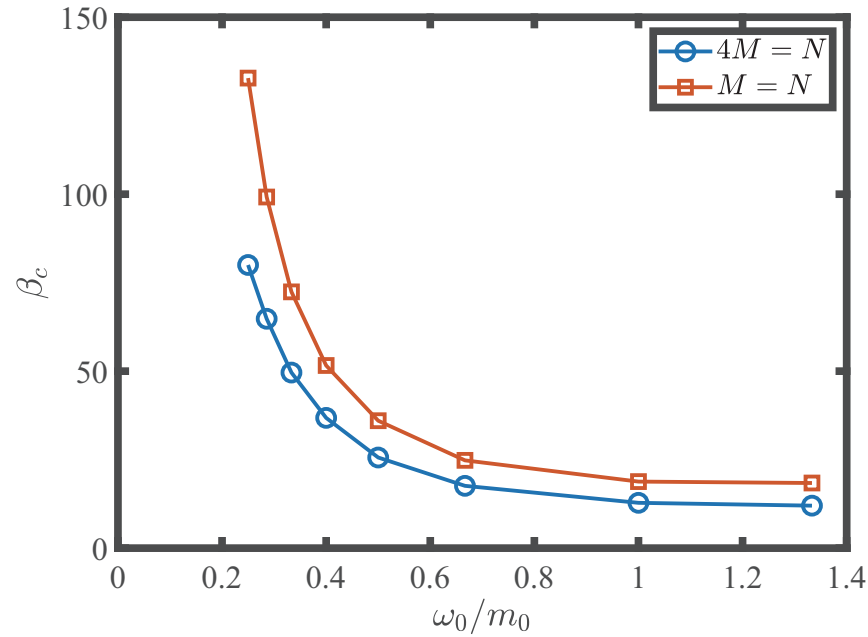

FIG. 3. Inverse transition temperature $\beta_{c}$ from NFL to superconductivity as a function of the ratio $\omega_{0} / m_{0}$ for $N=4 M$ and $N=M$, obtained from solving Eq. (10) at large $N$.

\section{B. Pairing at $N, M \rightarrow \infty$ : Mean-field theory}

It is straightforward to see that the leading pairing instability mediated by the critical boson mode is toward a spin-singlet, intradot, and intraflavor channel:

$$
\Delta \sim \sum_{i, \alpha}\left\langle c_{i \alpha \uparrow}^{\dagger} c_{i \alpha \downarrow}^{\dagger}\right\rangle .
$$

Within mean-field theory, the pairing behavior is described by the Eliashberg equation

$$
\Delta\left(i \omega_{n}\right)=\omega_{0}^{3} T \sum_{n} G_{b}\left(i \Omega_{n}\right)\left|G_{f}\left(i \omega_{m}+i \Omega_{n}\right)\right|^{2} \Delta\left(i \omega_{n}+i \Omega_{m}\right),
$$

where the $1 / M N$ factor given by the two Yukawa interaction vertices has been canceled by the summation of the site and flavor indices of the internal fermions.

At $T=0$, plugging in the analytical results in Eq. (3), we have

$$
\Delta(\omega)=\frac{2}{\alpha(x)} \int_{\Delta}^{\omega_{0}} \frac{d \omega^{\prime}}{2 \pi} \frac{\Delta\left(\omega^{\prime}\right)}{\left|\omega-\omega^{\prime}\right|^{1-2 x}\left|\omega^{\prime}\right|^{2 x}} .
$$

where $\Delta$ is the order of magnitude of the frequency-dependent gap $\Delta(\omega)$ that serves as an infrared cutoff of the Green's functions and $\omega_{0} \sim m_{0}$ is an ultraviolet cutoff scale for the low-energy quantum-critical NFL behavior.

At finite temperatures, we can solve for the critical temperatures $T_{c}$ using the normal-state results numerically obtained. To that end, we numerically solve Eq. (10) as an eigenvalue problem. As the temperature lowers, the eigenvalues of the kernel increase, and $T_{c}$ corresponds to the temperature at which the largest eigenvalue approaches 1. For reference, we plot $\beta_{c}\left(T_{c}=\frac{1}{\beta_{c}}\right)$ as a function of the ratio $\omega_{0} / m_{0}$ for $N=4 M$ and $N=M$ in Fig. 3. We see that as the dimensionless coupling constant $\omega_{0} / m_{0}$ decreases, $\beta_{c}$ increases $\left(T_{c}\right.$ decreases) in both cases. This is consistent with the fact that like $\omega_{0} / m_{0}$, the typical scale of the NFL behavior decreases as $\omega_{F} / \omega_{0} \sim\left(\omega_{0} / m_{0}\right)^{2}$, and that $T_{c} \lesssim \omega_{F}$ is an instability of the NFL. 

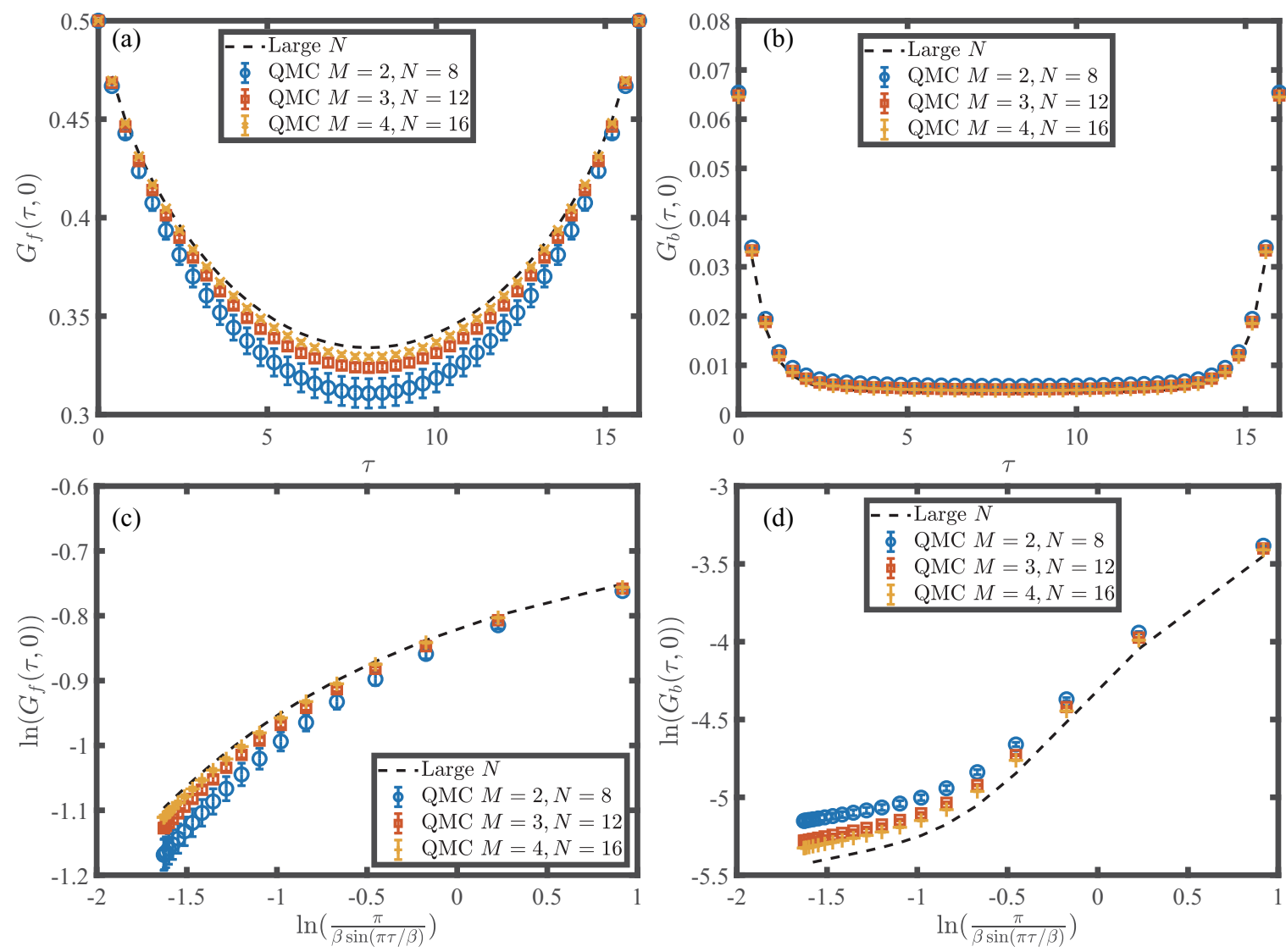

FIG. 4. QMC results at $N=4 M, m_{0}=2, \omega_{0}=1$, and $\beta=16$ for $M=2,3$, 4. (a) Green's function of fermions $G_{f}(\tau, 0)$ versus $\tau$ in the range of $\tau \in[0, \beta]$. Blue, red, and yellow dots are DQMC data, and the black dashed line is the large- $N$ result. (b) Green's function of bosons $G_{b}(\tau, 0)$ versus $\tau$ in the range of $\tau \in[0, \beta]$. (c) and (d) The same as above, but in a special log-log scale as in Fig. 2. The convergence towards the large- $N$ results as $(M, N)$ increase is obvious. In (a)-(d), the error bars denote the variation of the Green's function for different disorder realizations. The progressively smaller error bars as $M$ increases indicate that the randomness of the coupling self-averages.

At finite $N, M$, a true phase transition to a superconductor is absent. Yet, pairing fluctuations, which become stronger upon lowering temperatures, do contribute to the fermion Green's function, making the fermions more incoherent. In the Yukawa-SYK model, such effects are suppressed by $\mathcal{O}(1 / M N)$ but can be detected at small $M, N$ and at low temperatures where pairing susceptibility $\chi_{p} \gtrsim M N$. A true finite-temperature phase transition to superconductivity, on the other hand, can be obtained by a finite-size extrapolation of the pairing susceptibility in the QMC simulations. However, the calculation of such observables is beyond the scope of this work. In this paper, we focus on the NFL normal state, although we will discuss signatures of pairing fluctuations in $G_{f}$ obtained by QMC.

We end this section on large- $N$ results by summarizing the system behavior in three different regimes. At high temperatures $T \gg \omega_{F}$, the system behaves as trivial free fermions and bosons. At $T<\omega_{F}$ the system behaves as a quantumcritical NFL, with a pairing instability at $T_{c}<\omega_{F}$. Such a system behavior has also been discussed extensively in Ref. [36]. For finite $N$ the pairing transition does not occur, but pairing fluctuations become important in the low-temperature regime.

\section{NUMERICAL RESULTS}

\section{A. NFL Green's functions}

In this section, we report the key numerical findings in this paper, the NFL Green's function and self-tuned quantum criticality at finite values of $(M, N)$. We choose $\omega_{0}=1, m_{0}=2$, such that the dimensionless coupling $\omega_{0} / m_{0}$ is reasonably weak, and the pairing fluctuations discussed in Sec. II B do not significantly modify the normal-state NFL behavior.

Figure 4 demonstrates the fermion and boson Green's functions obtained in QMC simulation. We focus on $G_{b}$ and $G_{f}$ obtained with $N=4 M, \omega_{0}=1$, and $m_{0}=2$ at $\beta=16$ for $M=2, M=3$, and $M=4$, respectively. Each data point is obtained by averaging over 20 disorder realizations in $\left\{t_{i \alpha, j \beta}\right\}$. Figures 4(a) and 4(b) are plotted in linear scale, and one can see that the QMC curves are progressively close to the large- $N$ curve as $N$ increases. In Figs. 4(c) and 4(d), we present $G_{b}$ and $G_{f}$ versus $\pi /[\beta \sin (\pi \tau / \beta)]$ in a $\log -\log$ scale, as suggested in Eq. (8). It is clear that the QMC results match very well with the large- $N$ result and approach the latter as $N$ increases. The (rather small) error bars in Fig. 4 denote the variance of the QMC results with different realizations of random couplings [51]. We see that such variance decreases upon increasing $N$. 

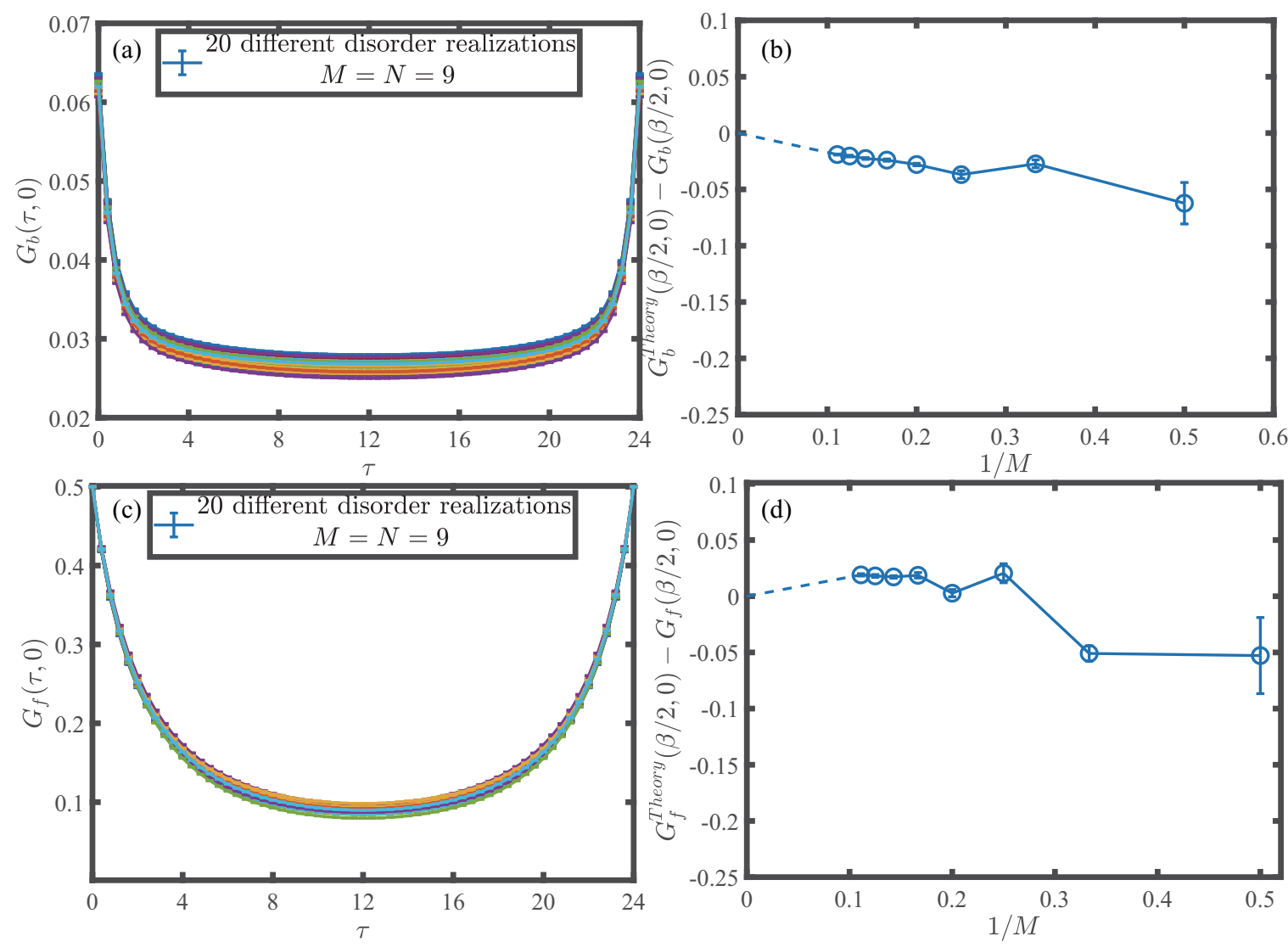

FIG. 5. (a) and (c) show $G_{b}$ and $G_{f}$ of 20 different disorder realizations with $M=N=9, \beta=24, m_{0}=2$, and $\omega_{0}=1$. The Green's functions are very close to each other. (b) and (d) present the difference between theoretical (large $N$ ) results $G^{\mathrm{Th}}(\beta / 2,0)$ and QMC numerical simulation data $G(\beta / 2,0)$. It is clear that as $M(N)$ increases, the distance between QMC and analytics gradually reduces. The relative standard deviations in the QMC data are also decreasing. The parameters are set at $M=N, \beta=24, m_{0}=2$, and $\omega_{0}=1$.

This is consistent with the self-averaging behavior of disordered systems and indicates that our values of $M=3, N=12$ and $M=4, N=16$ can be reasonably regarded as close to "large $N . "$

To further quantify the extrapolation to large $N$, we simulated the model for $N=M$; such a parameter choice allows us to go deeper into the $M$ sequence, and the smaller system size also allows us to go to lower temperatures. In Figs. 5(a) and 5(c), we plot the QMC data of $G_{b}(\tau, 0)$ and $G_{f}(\tau, 0)$ with $M=N=9, \beta=24, m_{0}=2$, and $\omega_{0}=1$, averaged over 20 disorder realizations. As before, we see the self-averaging behavior of disorder realizations. In Figs. 5(b) and 5(d), we plot the distance between the QMC disorder averaged Green's functions $G_{b}\left(\frac{\beta}{2}, 0\right)$ and $G_{f}\left(\frac{\beta}{2}, 0\right)$ and those from the large- $N$ analytical calculation $G_{b}^{\mathrm{Th}}\left(\frac{\beta}{2}, 0\right)$ and $G_{f}^{\mathrm{Th}}\left(\frac{\beta}{2}, 0\right)$. As $1 / N \rightarrow 0$, indeed $G_{b}\left(\frac{\beta}{2}, 0\right)$ approaches its large- $N$ value. This indicates that the replica-off-diagonal fluctuations are small and suppressed by $1 / N$. As a result, glass behavior is absent in this model at least down to $\beta=24$. In the meantime, $G_{f}\left(\frac{\beta}{2}, 0\right)$ is quite close to its large- $N$ value but remains slightly smaller up to $N=9$. Contrasting the behaviors of $G_{b}$ and $G_{f}$, it is tempting to attribute the deviation of $G_{f}$ to pairing fluctuations. This is consistent with the facts that pairing fluctuations makes the fermions more incoherent and that $T=1 / 24$ is quite close to the critical temperature $T_{c}=1 / 36$, as shown in Fig. 3 for the case of $N=M$. We expect that as $N$ further increases, the effect of pairing fluctuations will be suppressed and the pairing transition at $N=\infty$ is mean-field-like. A detailed study of the pairing transition is needed.

We emphasize that the randomness of the Yukawa coupling is crucial in stabilizing the NFL behavior. To demonstrate this, in Appendix $\mathrm{C}$ we consider a very similar model, in which the random coupling $t_{i j}$ is of lower rank and does not depend on $\alpha, \beta$. This model was analyzed by one of us [35] using the Schwinger-Dyson equation at large $N$, and the analytical results are practically identical to those here. However, our QMC studies have found that its low-temperature phase is actually a spin glass, as the bosonic Green's function has a large static component. Somewhat counterintuitively, the glass phase absent in our present model is realized in such a "less random" model. Indeed, one can show that in this model, certain replica-off-diagonal diagrams that are not suppressed by $1 / N$ survive the disorder averaging (see Appendix C), thanks to the lower-rank randomness in the Yukawa coupling, and are expected to drive the glass transition [52]. Therefore the random coupling taken in Ref. [35] needs to be modified to the present higher-rank form.

It is also interesting to investigate the evolution of finite$N$ corrections as a function of temperature. In the original SYK model, it is well known that the strength of replicaoff-diagonal fluctuations increases with lowering temperature 

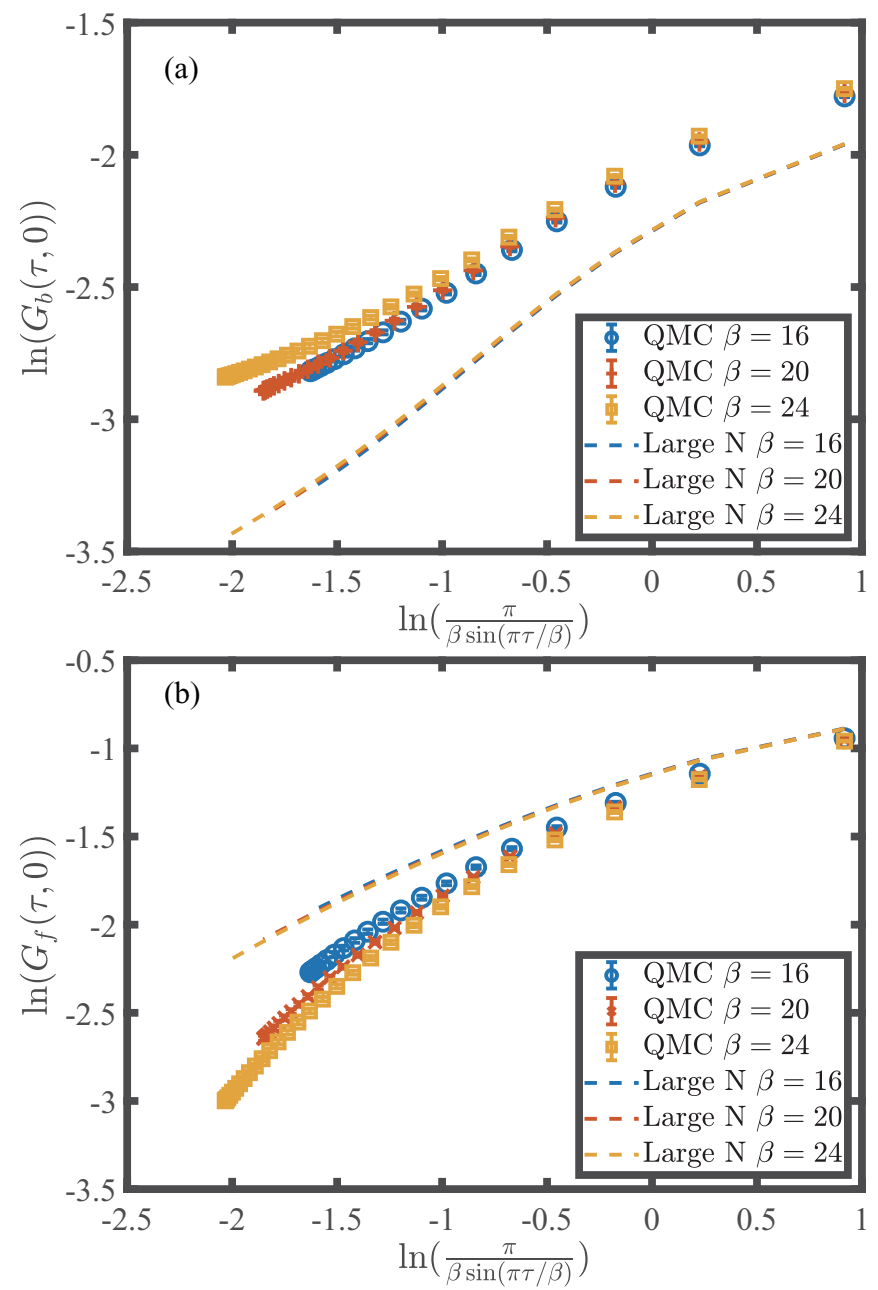

FIG. 6. QMC results of (a) $G_{b}$ and (b) $G_{f}$ at $M=4, N=16$, $m_{0}=\omega_{0}=1$, and $\beta=16,20,24$. In $\log$-log plots, we see that as $\beta$ increases, large- $N$ results are basically unchanged, while curves of the QMC simulation progressively deviate from the large- $N$ value due to increasing finite- $N$ corrections.

as $\sim 1 / N \log ^{2}(T)$, which has led to initial speculations of a glass transition at exponentially low temperatures. On the other hand, the strength of pairing fluctuations also increases with lowering temperature. To enable a clear analysis of the fluctuation effects, we numerically simulated the intermediate-coupling regime of our model with $\omega_{0}=m_{0}=$ 1. Shown in Fig. 6, we see that in this case the deviation between numerical and large- $N$ results is indeed more pronounced and increases upon lowering the temperature. Furthermore, the finite- $N$ corrections modify $G_{b}$ upward and $G_{f}$ downward. The upward deviation in $G_{b}$ is consistent with replica-off-diagonal fluctuations, since they make $G_{b}$ more static, just as in a glass transition. On the other hand, the downward deviation in $G_{f}$ is likely to predominantly come from pairing fluctuations.

\section{B. Self-tuned quantum criticality}

As discussed in the Sec. II A, the self-tuned quantum criticality occurs independently of the bare boson mass $m_{0}$, at least at the large- $N$ limit. We numerically tested this expecta- tion in QMC simulation with $M=4, N=16$. The results are shown in Fig. 7. As a comparison with our interacting model, Fig. 7(a) shows the bare boson Green's function generated from $H_{b}$ in Eq. (A5) where the mass is $m_{0}=1$ and $\beta=16$. With such a mass term, the Green's function clearly exhibits exponential decay in imaginary time to $G_{b}(\tau=\beta / 2,0) \approx 0$. We can see it from the log-log plot: At the far left of the curve $\tau \rightarrow \beta$, which corresponds to the long-time limit, the value of $\ln \left[G_{b}(\tau, 0)\right]$ decays rapidly. However, once coupled with fermions in our model, as shown in Figs. 7(b) $\left(m_{0}=\omega_{0}=\right.$ 1 , averaged over 20 realizations) and 7(c) $\left(m_{0}=2 \omega_{0}=2\right.$, averaged over 20 realizations), with different masses while keeping the $M=4, N=16$ and $\beta=16$, the boson Green's functions become critical. The Green's functions $G_{b}$ in imaginary time in both cases do not decay exponentially, but instead are well consistent with the power-law form of Eq. (8). In Figs. 7(b) and 7(c), besides the QMC data, we plotted a red dashed line, which is the large- $N$ result. The data in Fig. 7(c) turn out to be very close to the theoretical result. Remarkably, here we see that it does not require tuning the bare mass $m_{0}$ for the system to exhibit quantum-critical behavior, therefore exhibiting the self-tuned quantum criticality, consistent with analytical predictions at large $N$.

\section{DISCUSSION}

In this paper, we performed unbiased sign-problem-free quantum Monte Carlo simulations of the Yukawa-SYK model and reported direct evidence of self-tuned quantum-critical and NFL behaviors. We believe that such SYK-like models provide a new venue to construct analytical solvable models for strange metals and unconventional superconductors. Our work serves as a starting point to further analyze such models beyond the analytical large- $N$ limit, in a numerically unbiased manner. Further studies in several further directions are in order.

First, the numerical framework developed here allows one to incorporate the Hubbard $U$ interaction at half-filling without the fermion sign problem. From a theoretical point of view, such a generalized model likely exhibits a strange-metal to Mott insulator transition. It will also be interesting to study whether a spin-glass phase can be realized in between, resembling the phase diagram of the underdoped cuprates. Second, recent works have revealed exotic quantum phase transitions between a strange metal and a trivial insulator as one varies the filling [38], but analytical results have only been obtained in the weak-coupling limit. It is an open question whether more exotic phases exist at stronger coupling. Finally, the quantum dot model studied here can be generalized to a lattice model [53-55], in which more thermodynamical and transport properties can be examined.

In terms of numerical methodologies, the present work opens the directions of combining the randomness and allconnected models in the study of correlated electron systems, hence greatly broadening the scope of the correlated and itinerant systems. The Yukawa-SYK model and its QMC simulation provide a concrete example of NFL and give us the chance to have a systematic comparison with the large- $N$ analytical calculation. Therefore one can certainly foresee that more realistic and insightful NFL lattice models will 

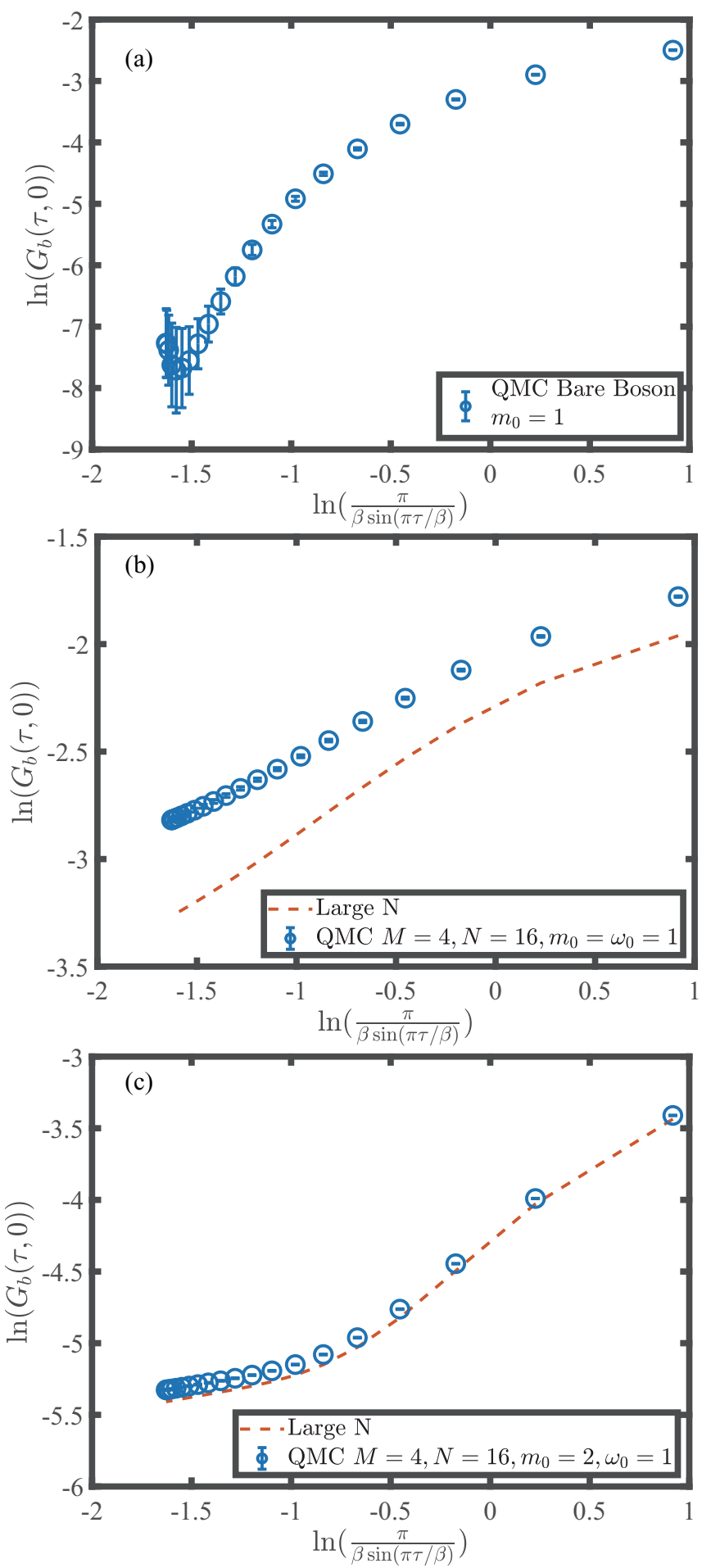

FIG. 7. Self-tuned quantum criticality with different boson masses in log-log plots. (a) $G_{b}(\tau, 0)$ from a free-boson model with $m_{0}=1$ and $\beta=16$. The exponential decay in imaginary time is evident with $\ln \left[G_{b}(\tau=\beta / 2)\right] \sim-7$. (b) and (c) show the $G_{b}(\tau, 0)$ from the Yukawa-SYK model in Eq. (1) with different mass $m_{0}=\omega_{0}$ (b) and $m_{0}=2 \omega_{0}$ (c) with $\omega_{0}=1$ at $M=4, N=16$ and $\beta=16$. Blue circles are DQMC data, and the red dashed lines are the large- $N$ result. Power-law decay of $G_{b}$ at low temperatures and long-time limit is clear to see in the log-log plots. These results reveal the self-tuned quantum criticality in our system. eventually be solved with unbiased quantum many-body numerics like the one presented here.

\section{ACKNOWLEDGMENTS}

We thank Yingfei Gu, Grigory Tarnopolsky, Subir Sachdev, and Steven Kivelson for insightful discussions. G.P. thanks Rui-Zhen Huang for helpful discussions on numerical calculations. G.P.P., W.W. and Z.Y.M. acknowledge support from the RGC of Hong Kong SAR China (Grants No. GRF 17303019 and No. 17301420), MOST through the National Key Research and Development Program (Grant No. 2016YFA0300502) and the Strategic Priority Research Program of the Chinese Academy of Sciences (Grant No. XDB33020300). We thank the Center for Quantum Simulation Sciences in the Institute of Physics, Chinese Academy of Sciences, the Computational Initiative at the Faculty of Science and the Information Technology Services at the University of Hong Kong and the Tianhe-1A, Tianhe-2, and Tianhe-3 prototype platforms at the National Supercomputer Centers in Tianjin and Guangzhou for their technical support and generous allocation of CPU time. This research was initiated at the Aspen Center for Physics, supported by NSF Grant No. PHY-1066293.

\section{APPENDIX A: DQMC METHODOLOGY}

The model described in Eq. (1) can be solved under the determinant quantum Monte Carlo (DQMC) framework [27,5659]. DQMC is the method of choice to study interacting electron systems and has been used extensively in the past few decades in addressing problems such as the Hubbard [58], $t-J$ [60], and Kondo lattice [61] models; lately, some great progress has been made in extending the DQMC scheme to interacting topological states of matter $[62,63]$, duality and QCP beyond the Landau-Ginzburg-Wilson paradigm [64,65], and, more relevant to this work, the designer Hamiltonians of critical bosons coupled to fermions via Yukawa interactions [23-25,27,29,30,47,49,66-68]. In this Appendix, we will elucidate the DQMC setting for the model in Eq. (1) in detail.

First, the partition function reads

$$
\begin{aligned}
Z & =\operatorname{Tr}\left\{e^{-\beta \hat{H}}\right\}=\operatorname{Tr}\left\{\left(e^{-\Delta \tau \hat{H}}\right)^{L_{\tau}}\right\} \\
& =\int\left(\prod_{\alpha \beta} d \phi_{\alpha \beta}\right) \operatorname{Tr}_{\mathbf{F}}\left\langle\phi_{11} \cdots \phi_{N N}\left|\left(e^{-\Delta \tau \hat{H}}\right)^{L_{\tau}}\right| \phi_{11} \cdots \phi_{N N}\right\rangle,
\end{aligned}
$$

where we divide the imaginary time axis into $L_{\tau}$ slices, $\beta=$ $L_{\tau} \times \Delta \tau$. Let the bosonic configuration at each time slice, $\vec{\Phi}_{l}=\left(\phi_{11, l}, \phi_{12, l}, \ldots, \phi_{N(N-1), l}, \phi_{N N, l}\right)$, serve as the complete basis of imaginary time propagation in the path integral; then

$$
\begin{aligned}
Z= & \int\left(\prod_{l=1}^{L_{\tau}} d \vec{\Phi}_{l}\right) \operatorname{Tr}_{\mathbf{F}}\left\langle\vec{\Phi}_{1}\left|e^{-\Delta \tau \hat{H}}\right| \vec{\Phi}_{L_{\tau}}\right\rangle\left\langle\vec{\Phi}_{L_{\tau}}\left|e^{-\Delta \tau \hat{H}}\right| \vec{\Phi}_{L_{\tau}-1}\right\rangle \\
& \cdots\left\langle\vec{\Phi}_{2}\left|e^{-\Delta \tau \hat{H}}\right| \vec{\Phi}_{1}\right\rangle .
\end{aligned}
$$


With the help of Suzuki-Trotter decomposition of the Hamiltonian in Eq. (1), one has

$$
e^{-\Delta \tau \hat{H}} \approx e^{-\Delta \tau \hat{H}_{f b}} e^{-\Delta \tau \hat{H}_{b}},
$$

where

$$
\begin{gathered}
H_{f b}=\sum_{i, j}^{M} \sum_{\alpha, \beta}^{N} \sum_{m, n}^{\uparrow \downarrow} \frac{i}{\sqrt{M N}} t_{i \alpha, j \beta} \phi_{\alpha \beta} c_{\alpha i m}^{\dagger} \sigma_{m, n}^{z} c_{\beta j n}, \\
H_{b}=\sum_{\alpha, \beta=1}^{N}\left(\frac{1}{2} \pi_{\alpha \beta}^{2}+\frac{m_{0}^{2}}{2} \phi_{\alpha \beta}^{2}\right)
\end{gathered}
$$

are the fermion-boson coupled term and the bosonic term, respectively.

\section{Bosonic part}

Sine we use the space-time arrangement of the bosons $\left\{\vec{\Phi}_{l}\right\}$ to span the configuration space, we need to first express the canonical momentum $\pi_{\alpha \beta}$ in Eq. (A5) in this configuration space. To this end, we first use the coherent state path integral

$$
\left|\phi_{\alpha \beta}\right\rangle=\frac{1}{\sqrt{2 \pi}} \int d \pi_{\alpha \beta} e^{-i \pi_{\alpha \beta} \phi_{\alpha \beta}}\left|\pi_{\alpha \beta}\right\rangle ;
$$

then the momentum term in the partition function can be expressed as

$$
\begin{aligned}
\left\langle\phi^{\prime}\left|e^{-\frac{1}{2} \Delta \tau \hat{\pi}^{2}}\right| \phi\right\rangle & =\frac{1}{2 \pi} \int d \pi e^{i \pi\left(\phi^{\prime}-\phi\right)-\frac{1}{2} \pi^{2} \Delta \tau} \\
& \simeq C e^{-\frac{\left(\phi^{\prime}-\phi\right)^{2}}{2 \Delta \tau}},
\end{aligned}
$$

where $C$ is a constant and $l$ and $l^{\prime}$ are two consecutive time slices along the imaginary time axis. The partition function then becomes

$$
\begin{aligned}
Z= & \int \prod_{l=1}^{L_{\tau}} d \vec{\Phi}_{l} \\
& \times \underbrace{C^{L_{\tau}}\left(\prod_{l=1}^{L_{\tau}} \prod_{\alpha, \beta=1}^{N} e^{-\Delta \tau \frac{m_{0}^{2}}{2} \phi_{\alpha \beta, l}^{2}}\right)\left(\prod_{\left\langle l, l^{\prime}\right\rangle} \prod_{\alpha, \beta=1}^{N} e^{-\frac{\left(\phi_{\alpha \beta, l}-\phi_{\alpha \beta,}, l^{\prime}\right)^{2}}{2 \Delta \tau}}\right)}_{\mathcal{W}_{b}} \\
& \times \underbrace{\operatorname{Tr}_{\mathbf{F}}\left\{e^{-\Delta \tau \hat{H}_{f b}\left(\vec{\Phi}_{L \tau}\right)} \cdots e^{-\Delta \tau \hat{H}_{f b}\left(\vec{\Phi}_{1}\right)}\right\}}_{\mathcal{W}_{f b}},
\end{aligned}
$$

where the first ( ) in $\mathcal{W}_{b}$ contains the spatial boson interaction and the second ( ) in $\mathcal{W}_{b}$ contains the temporal boson interaction, $\left\langle l, l^{\prime}\right\rangle$ stands for the nearest-neighbor interaction in the imaginary time direction, and the $\operatorname{Tr}_{\mathbf{F}}$ in $\mathcal{W}_{f b}$ is the fermion trace we will deal with in Appendix A 2. It is now clear that the Monte Carlo sampling is performed in the bosonic field $\{\vec{\Phi}\}$ space of dimension $N \times N \times L_{\tau}$ or $M N \times M N \times L_{\tau}$ if one considers the random hopping $t_{\alpha \beta}$ in $H_{f b}$, and the configurational weight is composed of the bosonic part $\mathcal{W}_{b}$ and the fermion determinant $\mathcal{W}_{f b}$.

\section{Fermion determinant}

For a specific bosonic configuration, the fermion determinant is of quadratic form and can be evaluated as that of the free system, following the standard expression

$$
\operatorname{Tr}_{\mathbf{F}}\left\{e^{-\sum_{i, j} \hat{c}_{i}^{\dagger} A_{i, j} \hat{c}_{j}} e^{-\sum_{i, j} \hat{c}_{i}^{\dagger} B_{i, j} \hat{c}_{j}}\right\}=\operatorname{Det}\left(\mathbf{I}+e^{-\mathbf{A}} e^{-\mathbf{B}}\right) .
$$

For the imaginary time propagation in the fermion trace in Eq. (A8), we define

$$
B\left(l_{2} \Delta \tau, l_{1} \Delta \tau\right)=\prod_{l=l_{1}+1}^{l_{2}} e^{-\Delta \tau V\left(\vec{\Phi}_{l}\right)},
$$

where

$$
V\left(\vec{\Phi}_{l}\right)=\frac{i}{\sqrt{M N}} \sigma_{2 \times 2}^{z} \otimes\left(t_{i \alpha, j \beta} \phi_{\alpha \beta, l}\right)_{M N \times M N} .
$$

It is interesting to note that in the conventional Hubbard-type model setting, there also exists a fermion hopping matrix on the exponential form, but since here we only have fermions Yukawa coupled with the bosonic field, the hopping matrix is reduced to an identical matrix, and the interaction matrix $V\left(\vec{\Phi}_{l}\right)$, which depends on the space-time configuration of the bosonic field $\left\{\vec{\Phi}_{l}\right\}$, contains both the randomness in the hopping matrix $\sigma_{2 \times 2}^{z} \otimes\left(t_{i, j}\right)_{M \times M}$ and the bosonic fluctuation matrix $\left(\phi_{\alpha \beta, l}\right)_{N \times N}$. In this way, after tracing out the fermion operators $c_{\alpha i m}^{\dagger}$ and $c_{\beta j n}$, the resulting fermion determinant is the determinant of matrices with size $M N \times M N$ and block diagonal in the fermion spin space of $\sigma^{z}$.

With these notations prepared, finally the partition function in Eq. (A8) can now be written as

$$
\begin{aligned}
Z= & \int \prod_{l=1}^{L_{\tau}} d \vec{\Phi}_{l} \\
& \times \underbrace{C^{L_{\tau}}\left(\prod_{l=1}^{L_{\tau}} \prod_{\alpha, \beta=1}^{N} e^{-\Delta \tau \frac{m_{0}^{2}}{2} \phi_{\alpha \beta, l}^{2}}\right)\left(\prod_{\left\langle l, l^{\prime}\right\rangle} \prod_{\alpha, \beta=1}^{N} e^{\left.-\frac{\left(\phi_{\alpha \beta, l}-\phi_{\alpha \beta, l}\right)^{2}}{2 \Delta \tau}\right)}\right)}_{\mathcal{W}_{b}} \\
& \times \underbrace{\operatorname{Det}\left[\mathbf{1}+B\left(L_{\tau} \Delta \tau,\left(L_{\tau}-1\right) \Delta \tau\right) \cdots B(\Delta \tau, 0)\right]}_{\mathcal{W}_{f b}} . \text { (A12) }
\end{aligned}
$$

This is the partition function describing the SYK-Yukawa model in Eq. (1), and we can now simulate it in DQMC.

\section{Free from sign problem}

As aforementioned, the partition function in Eq. (A12) is free from the minus-sign problem in the protection of a time-reversal symmetry [69]; that is, the Hamiltonian is invariant under such a symmetry operation. This can be easily demonstrated as follows.

First, we note that

$$
\begin{aligned}
H_{f b}= & \sum_{i, j=1}^{M} \sum_{\alpha, \beta=1}^{N} \frac{i}{\sqrt{M N}} t_{i \alpha, j \beta} \phi_{\alpha \beta} c_{\alpha i \uparrow}^{\dagger} c_{\beta j \uparrow} \\
& -\frac{i}{\sqrt{M N}} t_{i \alpha, j \beta} \phi_{\alpha \beta} c_{\alpha i \downarrow}^{\dagger} c_{\beta j \downarrow}
\end{aligned}
$$

and the time-reversal symmetry operator is $\mathcal{T}=i \sigma_{y} \mathcal{K}$. Its operation works as $\mathcal{T} c_{m} \mathcal{T}^{-1}=U_{m n} c_{n}, \mathcal{T} c_{m}^{\dagger} \mathcal{T}^{-1}=U_{m n}^{*} c_{n}^{\dagger}$, $\mathcal{T} i \mathcal{T}^{-1}=-i$, where $m, n=\uparrow / \downarrow, U=i \sigma_{y}$; then

$$
\mathcal{T} H_{f b} \mathcal{T}^{-1}=\sum_{i, j=1}^{M} \sum_{\alpha, \beta=1}^{N}-\frac{i}{\sqrt{M N}} t_{i \alpha, j \beta} \phi_{\alpha \beta} c_{\alpha i \downarrow}^{\dagger} c_{\beta j \downarrow}
$$




$$
+\frac{i}{\sqrt{M N}} t_{i \alpha, j \beta} \phi_{\alpha \beta} c_{\alpha i \uparrow}^{\dagger} c_{\beta j \uparrow}=H_{f b} .
$$

Therefore $H_{f b}$ is invariant under $\mathcal{T}$.

Next, notice that $V\left(\vec{\Phi}_{l}\right)$ is block diagonal in the space of $m, n=\uparrow, \downarrow$; then the fermion determinant can be written as

$$
\begin{aligned}
\operatorname{Det} & {[\mathbf{1}+B(\beta, 0)] } \\
& =\operatorname{Det}\left[\mathbf{1}+B^{\uparrow}(\beta, 0)\right] \operatorname{Det}\left[\mathbf{1}+B^{\downarrow}(\beta, 0)\right] \\
& =\operatorname{Det}\left[\mathbf{1}+B^{\uparrow}(\beta, 0)\right] \operatorname{Det}\left[\mathcal{T}\left(\mathbf{1}+B^{\downarrow}(\beta, 0)\right) \mathcal{T}^{-1}\right]^{*} \\
& =\operatorname{Det}\left[\mathbf{1}+B^{\uparrow}(\beta, 0)\right] \operatorname{Det}\left[\mathbf{1}+B^{\uparrow}(\beta, 0)\right]^{*} \\
& =\left|\operatorname{Det}\left[\mathbf{1}+B^{\uparrow}(\beta, 0)\right]\right|^{2},
\end{aligned}
$$

and it is positive definite. Also note that the boson weight $\mathcal{W}_{b}$ is positive definite as $\{\vec{\Phi}\}$ is the eigenstate of $H_{b}$ in space-time. So the entire configurational weight is positive definite, and there is no sign problem for the simulation.

\section{Update and measurement}

Another important ingredient in any Monte Carlo simulation is the update scheme between configurations; here, since the bosonic fields are continuous variables, we have to adapt to the local update with the Metropolis-type acceptance rate.

The ensemble average of physical observables can be expressed as

$$
\langle\hat{O}\rangle=\frac{\operatorname{Tr}\left\{e^{-\beta \hat{H}} \hat{O}\right\}}{\operatorname{Tr}\left\{e^{-\beta \hat{H}}\right\}}=\int\left(\prod_{l=1}^{L_{\tau}} d \vec{\Phi}_{l}\right) \mathcal{P}_{\mathcal{C}}\langle\hat{O}\rangle_{\mathcal{C}}+O\left(\Delta \tau^{2}\right),
$$

where $\Delta \tau^{2}$ systematical error comes from the Trotter decomposition and the weight and expectation value for each bosonic field configuration $\mathcal{C}$ are

$$
\begin{gathered}
\mathcal{P}_{\mathcal{C}}=\frac{\mathcal{W}_{\mathcal{C}}^{f b} \operatorname{Det}\left[1+B_{\mathcal{C}}(\beta, 0)\right]}{\int\left(\prod_{l=1}^{L_{\tau}} d \vec{\Phi}_{l}\right) \mathcal{W}_{\mathcal{C}}^{f b} \operatorname{Det}\left[1+B_{\mathcal{C}}(\beta, 0)\right]}, \\
\langle\hat{O}\rangle_{\mathcal{C}}=\frac{\operatorname{Tr}\left\{\hat{U}_{\mathcal{C}}(\beta, \tau) \hat{O} \hat{U}_{\mathcal{C}}(\tau, 0)\right\}}{\operatorname{Tr}\left\{\hat{U}_{\mathcal{C}}(\beta, 0)\right\}},
\end{gathered}
$$

where

$$
\hat{U}\left(l_{2} \Delta \tau, l_{1} \Delta \tau\right)=\prod_{l=l_{1}+1}^{l_{2}} e^{-\Delta \tau \hat{\mathbf{c}}^{\dagger} V\left(\vec{\Phi}_{l}\right) \hat{\mathbf{c}}} .
$$

Here, $\hat{\mathbf{c}}$ has $2 \times M \times N$ components, and so does the dimension of matrix $V$. After tracing out the quadratic fermions $\hat{\mathbf{c}}$ in Eq. (A19), one arrives at the $B\left(l_{2} \Delta \tau, l_{1} \Delta \tau\right)$ matrix in Eq. (A10), and the evaluation of the fermion determinant follows from there down to Eq. (A12). The detailed derivation of physical observables, exemplified by the equal time and imaginary time displaced fermionic Green's functions, is given in Appendix B.

Moreover, since the coupling matrix $t_{i j}$ in $H_{f b}$ is subject to randomness, the aforementioned Monte Carlo sample is performed for each disorder realization. Therefore, besides the Monte Carlo average over a fixed disorder configuration, the final physical observables such as the fermion and boson Green's functions are the disordered averaged quantities.

\section{APPENDIX B: MONTE CARLO MEASUREMENTS}

The ensemble average of physical observables, in the DQMC formalism, can be calculated as

$$
\begin{aligned}
\langle\hat{O}\rangle_{\mathcal{C}} & =\left.\frac{\partial}{\partial \eta} \ln \operatorname{Tr}\left[\hat{U}_{\mathcal{C}}(\beta, \tau) e^{\eta \hat{O}} \hat{U}_{\mathcal{C}}(\tau, 0)\right]\right|_{\eta=0} \\
& =\left.\frac{\partial}{\partial \eta} \ln \operatorname{Det}\left[\mathbf{1}+B_{\mathcal{C}}(\beta, \tau) e^{\eta O} B_{\mathcal{C}}(\tau, 0)\right]\right|_{\eta=0} \\
& =\left.\frac{\partial}{\partial \eta} \operatorname{Tr} \ln \left[\mathbf{1}+B_{\mathcal{C}}(\beta, \tau) e^{\eta O} B_{\mathcal{C}}(\tau, 0)\right]\right|_{\eta=0} \\
& =\operatorname{Tr}\left[B_{\mathcal{C}}(\tau, 0)\left(1+B_{\mathcal{C}}(\beta, 0)\right)^{-1} B_{\mathcal{C}}(\beta, \tau) O\right] \\
& =\operatorname{Tr}\left[\left(1-\left(1+B_{\mathcal{C}}(\tau, 0) B_{\mathcal{C}}(\beta, \tau)\right)^{-1}\right) O\right]
\end{aligned}
$$

in the case of the equal time fermionic Green's function, $\hat{O}=\hat{\mathbf{c}}^{\dagger} O \hat{\mathbf{c}}$. $\hat{U}_{\mathcal{C}}$ and $B_{\mathcal{C}}$ are defined in Eqs. (A19) and (A10), respectively.

For the imaginary time displaced fermionic Green's function, $G_{f, i j}(\tau, 0)=\left\langle c_{i}(\tau) c_{j}^{\dagger}(0)\right\rangle$, where $i, j$ encapsulate the dot flavor and spin indices in the Hamiltonian in Eq. (1) and the imaginary time $\tau \in[0, \beta]$, it can be evaluated in DQMC as

$$
\begin{aligned}
\left\langle c_{i}(\tau) c_{j}^{\dagger}(0)\right\rangle & =\frac{\operatorname{Tr}\left\{\hat{U}_{\mathcal{C}}(\beta, \tau) \hat{c}_{i} \hat{U}_{\mathcal{C}}(\tau, 0) \hat{c}_{j}^{\dagger}\right\}}{\operatorname{Tr}\left\{\hat{U}_{\mathcal{C}}(\beta, 0)\right\}} \\
& =\frac{\operatorname{Tr}\left\{\hat{U}_{\mathcal{C}}(\beta, 0)\left[\hat{U}_{\mathcal{C}}^{-1}(\tau, 0) \hat{c}_{i} \hat{U}_{\mathcal{C}}(\tau, 0)\right] \hat{c}_{j}^{\dagger}\right\}}{\operatorname{Tr}\left\{\hat{U}_{\mathcal{C}}(\beta, 0)\right\}} \\
& =\sum_{k} B_{\mathcal{C}}(\tau, 0)_{i k} \frac{\operatorname{Tr}\left\{\hat{U}_{\mathcal{C}}(\beta, 0) \hat{c}_{k} \hat{c}_{j}^{\dagger}\right\}}{\operatorname{Tr}\left\{\hat{U}_{\mathcal{C}}(\beta, 0)\right\}} \\
& =\left[B_{\mathcal{C}}(\tau, 0)\left(1+B_{\mathcal{C}}(\beta, 0)\right)^{-1}\right]_{i j},
\end{aligned}
$$

where the intermediate steps in Eq. (B2) are given explicitly in Ref. [58].

\section{APPENDIX C: SPIN-GLASS BEHAVIOR IN A LESS-RANDOM MODEL}

We construct a model in a similar form in which the random coupling is of a lower rank,

$$
\begin{aligned}
H= & \sum_{i, j=1}^{M} \sum_{\alpha, \beta=1}^{N} \sum_{m, n}^{\uparrow, \downarrow}\left(\frac{i}{\sqrt{M N}} t_{i, j} \phi_{\alpha \beta} c_{i \alpha ; m}^{\dagger} \sigma_{m, n}^{z} c_{j \beta ; n}\right) \\
& +\sum_{\alpha, \beta=1}^{N}\left(\frac{1}{2} \pi_{\alpha \beta}^{2}+\frac{m_{0}^{2}}{2} \phi_{\alpha \beta}^{2}\right),
\end{aligned}
$$

where the random coupling between fermion and boson is realized as $\left\langle t_{i j}\right\rangle=0,\left\langle t_{i j} t_{k l}\right\rangle=\left(\delta_{i k} \delta_{j l}+\delta_{i l} \delta_{j k}\right) \omega_{0}^{3}$. Still, for the sake of simplicity we set $\omega_{0}=1$ as the energy unit throughout the paper, and the temperature scale is then $T \equiv \omega_{0} / \beta . \pi_{\alpha \beta}$ is the canonical momentum of $\phi_{\alpha \beta}$. Hermiticity of the first term requires $\phi_{\alpha \beta}=-\phi_{\beta \alpha}$.

In Fig. 8, we show the static component (with $\omega_{n}=0$ ) for bosonic Green's function $G_{b}\left(\omega_{n}\right)$. In the large- $N$ limit, this component can be regarded as an Edwards-Anderson order parameter of the spin-glass phase [45]. As $N$ increases, the static component, along with its variance for different disorder realizations, increases with the increase in $N$ at 


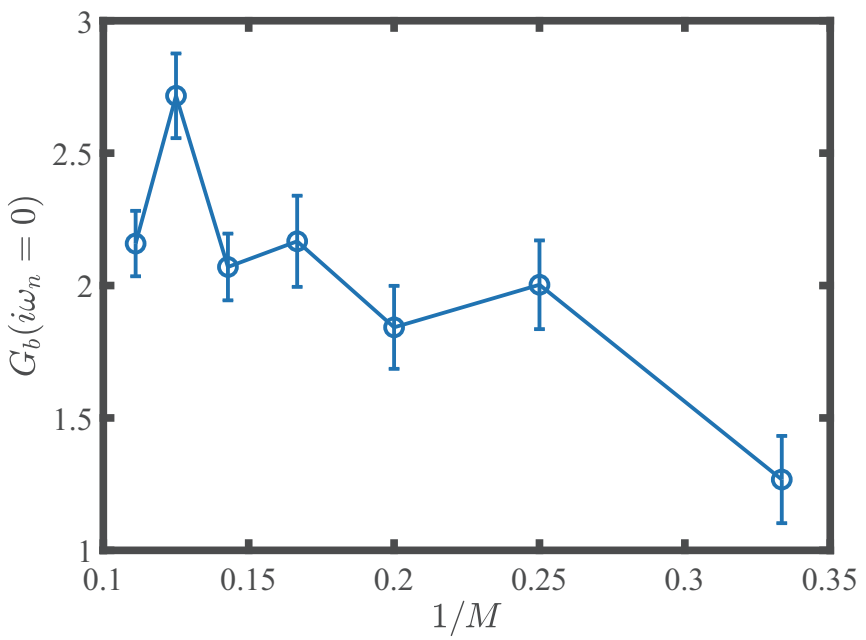

FIG. 8. DQMC results of Matsubara Green's function $G_{b}$ at $M=$ $N, m_{0}=\omega_{0}=1$, and $\beta=16$. We plot the zero-frequency component and its error bars. Glassy behaviors are seen.

$M=N, \beta=16$, and $m_{0}=\omega_{0}=1$, which is indicative of spin-glass behavior.

To analytically understand the difference between the models in Eqs. (C1) and (1), we find that due to the lower-rank random coupling in Eq. (C1), certain replica-offdiagonal fluctuations are not suppressed by large $M, N$. At low

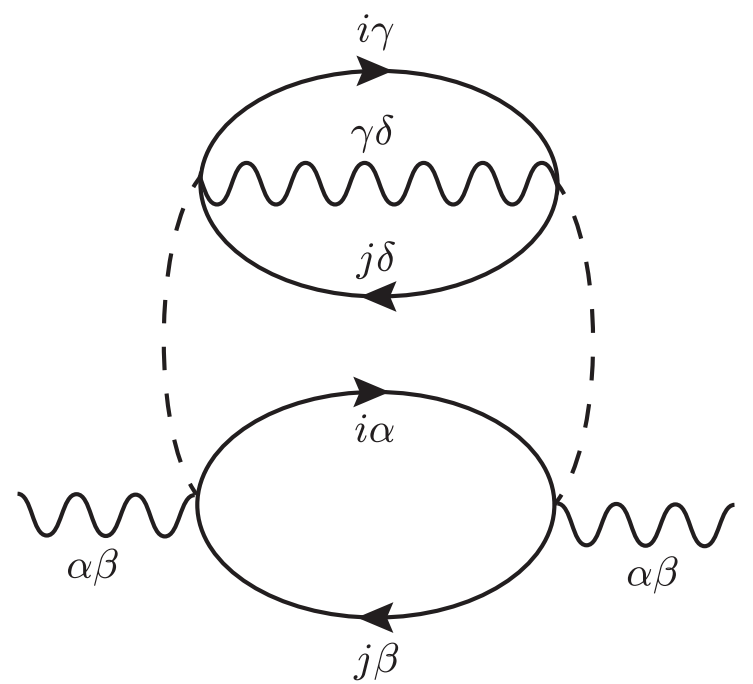

FIG. 9. A Feynman diagram involving replica-off-diagonal processes that are not suppressed by $1 / M N$ in the model given by Eq. (C1). The two fermion loops carry different replicon indices.

temperatures, these processes are responsible for driving a spin-glass transition and developing a replica-off-diagonal Edwards-Anderson order parameter. We show an example of such a diagram in Fig. 9; a systematic study of the glass transition is given in Ref. [52]. Such processes vanish in the large- $M, N$ limit of the model (1) studied in the main text.
[1] G. R. Stewart, Non-Fermi-liquid behavior in $d$ - and $f$-electron metals, Rev. Mod. Phys. 73, 797 (2001).

[2] A. Abanov, A. Chubukov, and J. Schmalian, Quantum-critical theory of the spin-fermion model and its application to cuprates: Normal state analysis, Adv. Phys. 52, 119 (2003).

[3] B. Keimer, S. A. Kivelson, M. R. Norman, S. Uchida, and J. Zaanen, From quantum matter to high-temperature superconductivity in copper oxides, Nature (London) 518, 179 (2015).

[4] Z. Liu, Y. Gu, W. Zhang, D. Gong, W. Zhang, T. Xie, X. Lu, X. Ma, X. Zhang, R. Zhang, J. Zhu, C. Ren, L. Shan, X. Qiu, P. Dai, Y.-f. Yang, H. Luo, and S. Li, Nematic Quantum Critical Fluctuations in $\mathrm{BaFe}_{2-x} \mathrm{Ni}_{x} \mathrm{As}_{2}$, Phys. Rev. Lett. 117, 157002 (2016).

[5] Y. Gu, Z. Liu, T. Xie, W. Zhang, D. Gong, D. Hu, X. Ma, C. Li, L. Zhao, L. Lin, Z. Xu, G. Tan, G. Chen, Z. Y. Meng, Y.-f. Yang, H. Luo, and S. Li, Unified Phase Diagram for Iron-Based Superconductors, Phys. Rev. Lett. 119, 157001 (2017).

[6] J. Custers, P. Gegenwart, H. Wilhelm, K. Neumaier, Y. Tokiwa, O. Trovarelli, C. Geibel, F. Steglich, C. Pépin, and P. Coleman, The break-up of heavy electrons at a quantum critical point, Nature (London) 424, 524 (2003).

[7] B. Shen, Y. Zhang, Y. Komijani, M. Nicklas, R. Borth, A. Wang, Y. Chen, Z. Nie, R. Li, X. Lu, H. Lee, M. Smidman, F. Steglich, P. Coleman, and H. Yuan, Strange metal behavior in a pure ferromagnetic Kondo lattice, Nature (London) 579, 51 (2020).

[8] Y. Cao, D. Chowdhury, D. Rodan-Legrain, O. Rubies-Bigorda, K. Watanabe, T. Taniguchi, T. Senthil, and P. Jarillo-Herrero,
Strange Metal in Magic-Angle Graphene with Near Planckian Dissipation, Phys. Rev. Lett. 124, 076801 (2020).

[9] C. Shen, Y. Chu, Q. Wu, N. Li, S. Wang, Y. Zhao, J. Tang, J. Liu, J. Tian, K. Watanabe, T. Taniguchi, R. Yang, Z. Y. Meng, D. Shi, O. V. Yazyev, and G. Zhang, Correlated states in twisted double bilayer graphene, Nat. Phys. 16, 520 (2020).

[10] S. Sachdev and J. Ye, Gapless Spin-Fluid Ground State in a Random Quantum Heisenberg Magnet, Phys. Rev. Lett. 70, 3339 (1993).

[11] A. Kitaev, A simple matter of quantum holography, in Entanglement in Strongly-Correlated Quantum Matter (April 6 to July 2, 2015), Talks at KITP, University of California, Santa Barbara, https://online.kitp.ucsb.edu/online/entangled15/.

[12] S. Sachdev, Bekenstein-Hawking Entropy and Strange Metals, Phys. Rev. X 5, 041025 (2015).

[13] A. Kitaev and S. J. Suh, The soft mode in the sSachdev-YeKitaev model and its gravity dual, J. High Energy Phys. 05 (2018) 183.

[14] H. Guo, Y. Gu, and S. Sachdev, Linear in temperature resistivity in the limit of zero temperature from the time reparameterization soft mode, Ann. Phys. (Amsterdam) 418, 168202 (2020).

[15] Y. Gu, X.-L. Qi, and D. Stanford, Local criticality, diffusion and chaos in generalized Sachdev-Ye-Kitaev models, J. High Energy Phys. 05 (2017) 125.

[16] M. A. Metlitski and S. Sachdev, Quantum phase transitions of metals in two spatial dimensions. I. Ising-nematic order, Phys. Rev. B 82, 075127 (2010). 
[17] M. A. Metlitski and S. Sachdev, Quantum phase transitions of metals in two spatial dimensions. II. Spin density wave order, Phys. Rev. B 82, 075128 (2010).

[18] M. A. Metlitski, D. F. Mross, S. Sachdev, and T. Senthil, Cooper pairing in non-Fermi liquids, Phys. Rev. B 91, 115111 (2015).

[19] S. Raghu, G. Torroba, and H. Wang, Metallic quantum critical points with finite BCS couplings, Phys. Rev. B 92, 205104 (2015).

[20] S. Lederer, Y. Schattner, E. Berg, and S. A. Kivelson, Enhancement of Superconductivity near a Nematic Quantum Critical Point, Phys. Rev. Lett. 114, 097001 (2015).

[21] M. J. Lawler, D. G. Barci, V. Fernández, E. Fradkin, and L. Oxman, Nonperturbative behavior of the quantum phase transition to a nematic Fermi fluid, Phys. Rev. B 73, 085101 (2006).

[22] M. J. Lawler and E. Fradkin, Local quantum criticality at the nematic quantum phase transition, Phys. Rev. B 75, 033304 (2007).

[23] X. Y. Xu, K. Sun, Y. Schattner, E. Berg, and Z. Y. Meng, Non-Fermi Liquid at $(2+1) D$ Ferromagnetic Quantum Critical Point, Phys. Rev. X 7, 031058 (2017).

[24] Z. H. Liu, X. Y. Xu, Y. Qi, K. Sun, and Z. Y. Meng, Itinerant quantum critical point with frustration and a non-Fermi liquid, Phys. Rev. B 98, 045116 (2018).

[25] Z. H. Liu, G. Pan, X. Y. Xu, K. Sun, and Z. Y. Meng, Itinerant quantum critical point with fermion pockets and hotspots, Proc. Natl. Acad. Sci. USA 116, 16760 (2019).

[26] X. Y. Xu, Y. Qi, L. Zhang, F. F. Assaad, C. Xu, and Z. Y. Meng, Monte Carlo Study of Lattice Compact Quantum Electrodynamics with Fermionic Matter: The Parent State of Quantum Phases, Phys. Rev. X 9, 021022 (2019).

[27] X. Y. Xu, Z. H. Liu, G. Pan, Y. Qi, K. Sun, and Z. Y. Meng, Revealing fermionic quantum criticality from new Monte Carlo techniques, J. Phys.: Condens. Matter 31, 463001 (2019).

[28] S.-S. Lee, Low-energy effective theory of Fermi surface coupled with $\mathrm{U}(1)$ gauge field in $2+1$ dimensions, Phys. Rev. B 80, 165102 (2009).

[29] E. Berg, M. Metlitski, and S. Sachdev, Sign-problem-free quantum Monte Carlo of the onset of antiferromagnetism in metals, Science 338, 1606 (2012).

[30] E. Berg, S. Lederer, Y. Schattner, and S. Trebst, Monte Carlo studies of quantum critical metals, Annu. Rev. Condens. Matter Phys. 10, 63 (2019).

[31] Y. Schattner, S. Lederer, Steven A. Kivelson, and E. Berg, Ising Nematic Quantum Critical Point in a Metal: A Monte Carlo Study, Phys. Rev. X 6, 031028 (2016).

[32] X. Y. Xu, A. Klein, K. Sun, Andrey V. Chubukov, and Z. Y. Meng, Identification of non-Fermi liquid fermionic self-energy from quantum Monte Carlo data, npj Quantum Mater. 5, 65 (2020).

[33] C. Chen, X. Y. Xu, Y. Qi, and Z. Y. Meng, Metal to orthogonal metal transition, Chin. Phys. Lett. 37, 047103 (2020).

[34] C. Chen, T. Yuan, Y. Qi, and Z. Y. Meng, Doped orthogonal metals become Fermi arcs, arXiv:2007.05543.

[35] Y. Wang, Solvable Strong-Coupling Quantum-Dot Model with a Non-Fermi-Liquid Pairing Transition, Phys. Rev. Lett. 124, 017002 (2020).

[36] I. Esterlis and J. Schmalian, Cooper pairing of incoherent electrons: An electron-phonon version of the Sachdev-Ye-Kitaev model, Phys. Rev. B 100, 115132 (2019).
[37] D. Hauck, M. J. Klug, I. Esterlis, and J. Schmalian, Eliashberg equations for an electron-phonon version of the Sachdev-YeKitaev model: Pair breaking in non-Fermi liquid superconductors, Ann. Phys. (Amsterdam) 417, 168120 (2020).

[38] Y. Wang and A. V. Chubukov, Quantum phase transition in the Yukawa-SYK model, Phys. Rev. Research 2, 033084 (2020).

[39] R. Bistritzer and A. H. MacDonald, Moiré bands in twisted double-layer graphene, Proc. Natl. Acad. Sci. USA 108, 12233 (2011).

[40] Y. Da Liao, J. Kang, Clara N. Breiø, X. Y. Xu, H.-Q. Wu, B. M. Andersen, R. M. Fernandes, and Z. Y. Meng, Correlationinduced insulating topological phases at charge neutrality in twisted bilayer graphene, arXiv:2004.12536.

[41] Y. Da Liao, X. Y. Xu, Z. Y. Meng, and J. Kang, Correlated insulating phases in the twisted bilayer graphene, Chin. Phys. B 30, 17305 (2021).

[42] J. Kim, X. Cao, and E. Altman, Low-rank Sachdev-Ye-Kitaev models, Phys. Rev. B 101, 125112 (2020).

[43] C. L. Baldwin and B. Swingle, Quenched vs Annealed: Glassiness from SK to SYK, Phys. Rev. X 10, 031026 (2020).

[44] A. Georges, O. Parcollet, and S. Sachdev, Quantum fluctuations of a nearly critical Heisenberg spin glass, Phys. Rev. B 63, 134406 (2001).

[45] W. Fu and S. Sachdev, Numerical study of fermion and boson models with infinite-range random interactions, Phys. Rev. B 94, 035135 (2016).

[46] G. Gur-Ari, R. Mahajan, and A. Vaezi, Does the SYK model have a spin glass phase? J. High Energy Phys. 11 (2018) 070 .

[47] M. H. Gerlach, Y. Schattner, E. Berg, and S. Trebst, Quantum critical properties of a metallic spin-density-wave transition, Phys. Rev. B 95, 035124 (2017).

[48] A. Klein, A. V. Chubukov, Y. Schattner, and E. Berg, Normal State Properties of Quantum Critical Metals at Finite Temperature, Phys. Rev. X 10, 031053 (2020).

[49] X. Wang, Y. Schattner, E. Berg, and R. M. Fernandes, Superconductivity mediated by quantum critical antiferromagnetic fluctuations: The rise and fall of hot spots, Phys. Rev. B 95, 174520 (2017).

[50] The Fourier transform from $\Pi(\Omega)$ is tricky, since the positive power-law $|\Omega|^{1-2 x}$ does not have a Fourier transform in the common sense as the Fourier integral is UV divergent for $0<$ $x<1 / 2$. This divergence is canceled by the Fourier transform of the $m_{0}^{2}$ term in $\Pi(\Omega)$ with the UV information.

[51] Notice that this is not to be confused with the inherent error of the QMC simulations, whose magnitude is much smaller than the variance from disorder.

[52] Y. Wang (unpublished).

[53] X.-Y. Song, C.-M. Jian, and L. Balents, Strongly Correlated Metal Built from Sachdev-Ye-Kitaev Models, Phys. Rev. Lett. 119, 216601 (2017).

[54] Z. Bi, C.-M. Jian, Y.-Z. You, K. A. Pawlak, and C. Xu, Instability of the non-Fermi-liquid state of the Sachdev-Ye-Kitaev model, Phys. Rev. B 95, 205105 (2017).

[55] D. Chowdhury, Y. Werman, E. Berg, and T. Senthil, Translationally Invariant Non-Fermi-Liquid Metals with Critical Fermi Surfaces: Solvable Models, Phys. Rev. X 8, 031024 (2018). 
[56] R. Blankenbecler, D. J. Scalapino, and R. L. Sugar, Monte Carlo calculations of coupled boson-fermion systems. I, Phys. Rev. D 24, 2278 (1981).

[57] J. E. Hirsch, Discrete Hubbard-Stratonovich transformation for fermion lattice models, Phys. Rev. B 28, 4059 (1983).

[58] J. E. Hirsch, Two-dimensional Hubbard model: Numerical simulation study, Phys. Rev. B 31, 4403 (1985).

[59] F. F. Assaad and H. G. Evertz, World-line and determinantal quantum Monte Carlo methods for spins, phonons and electrons, in Computational Many-Particle Physics, edited by $\mathrm{H}$. Fehske, R. Schneider, and A. Weiße, Lecture Notes in Physics Vol. 739 (Springer, Berlin, 2008), pp. 277-356.

[60] M. Brunner, F. F. Assaad, and A. Muramatsu, Single-hole dynamics in the $t-J$ model on a square lattice, Phys. Rev. B 62 , 15480 (2000).

[61] F. F. Assaad, Quantum Monte Carlo Simulations of the HalfFilled Two-Dimensional Kondo Lattice Model, Phys. Rev. Lett. 83, 796 (1999).

[62] M. Hohenadler, Z. Y. Meng, T. C. Lang, S. Wessel, A. Muramatsu, and F. F. Assaad, Quantum phase transitions in the Kane-Mele-Hubbard Model, Phys. Rev. B 85, 115132 (2012).

[63] Z. Y. Meng, H.-H. Hung, and T. C. Lang, The characterization of topological properties in quantum Monte Carlo simulations of the Kane-Mele-Hubbard model, Mod. Phys. Lett. B 28, 1430001 (2014).

[64] Y.-Y. He, H.-Q. Wu, Y.-Z. You, C. Xu, Z. Y. Meng, and Z.-Y. $\mathrm{Lu}$, Bona fide interaction-driven topological phase transition in correlated symmetry-protected topological states, Phys. Rev. B 93, 115150 (2016).

[65] Y. Q. Qin, Y.-Y. He, Y.-Z. You, Z.-Y. Lu, A. Sen, A. W. Sandvik, C. Xu, and Z. Y. Meng, Duality between the Deconfined Quantum-Critical Point and the Bosonic Topological Transition, Phys. Rev. X 7, 031052 (2017).

[66] Y. Liu, W. Wang, K. Sun, and Z. Y. Meng, Designer Monte Carlo simulation for the Gross-Neveu-Yukawa transition, Phys. Rev. B 101, 064308 (2020).

[67] C. Bauer, Y. Schattner, S. Trebst, and E. Berg, Hierarchy of energy scales in an $\mathrm{O}(3)$ symmetric antiferromagnetic quantum critical metal: A Monte Carlo study, Phys. Rev. Research 2, 023008 (2020).

[68] Y. Liu, Z. Y. Meng, and S. Yin, Fermion enhanced firstorder phase transition and chiral Gross-Neveu tricritical point, arXiv:2012.00449.

[69] C. Wu and S.-C. Zhang, Sufficient condition for absence of the sign problem in the fermionic quantum Monte Carlo algorithm, Phys. Rev. B 71, 155115 (2005). 